\title{
The lumen-apposing metal stent (LAMS)-in-LAMS technique as an intraprocedural rescue treatment during endoscopic ultrasound-guided gastroenterostomy
}

Endoscopic ultrasound-guided gastroenterostomy (EUS-GE) is a new effective mini-invasive treatment for gastric outlet obstruction (GOO) [1 - 3]. An anastomosis is created between the stomach and a duodenal (or jejunal) loop by placement of a lumen-apposing metal stent (LAMS). Stent displacement can be a serious complication, often requiring surgery.

We present the case of a 78-year-old man with $\mathrm{GOO}$ due to a metastatic adenocarcinoma of the duodenal bulb. Following obstruction of a previously placed duodenal metal stent, the patient was scheduled for an EUS-GE. With the patient under general anesthesia, a 20-mm dilation balloon (CRE; Boston Scientific) was passed over the wire through the stenosis into the jejunum under fluoroscopic guidance. Contrast medium with saline was injected through the balloon catheter to identify the target loop ( $\triangleright$ Fig. 1), which was then accessed with the electrocautery-enhanced delivery system of a 20-mm LAMS (AXIOS-EC; Boston Scientific) ( Video 1). A 0.035-inch guidewire was inserted through the LAMS delivery system to secure access to the jejunal loop, and the stent was released. On release, the distal flange dislodged from the jejunal loop into the peritoneum; however, the guidewire was still in place ( Fig.2). Suction was applied to maintain apposition of the target loop with the stomach while a second $20-\mathrm{mm}$ LAMS was successfully placed over the guidewire through the first LAMS ( Fig.3). The LAMS-in-LAMS system was then dilated with a balloon up to $20 \mathrm{~mm}$. Screening with contrast medium passed through the stents revealed no evidence of leakage.

If a wire access to the target loop is not preserved, LAMS misdeployment can require a natural orifice transluminal endoscopic surgery (NOTES)-based approach [4] or surgery. Wire access should therefore always be preserved to allow a minimally invasive rescue approach [5]. The
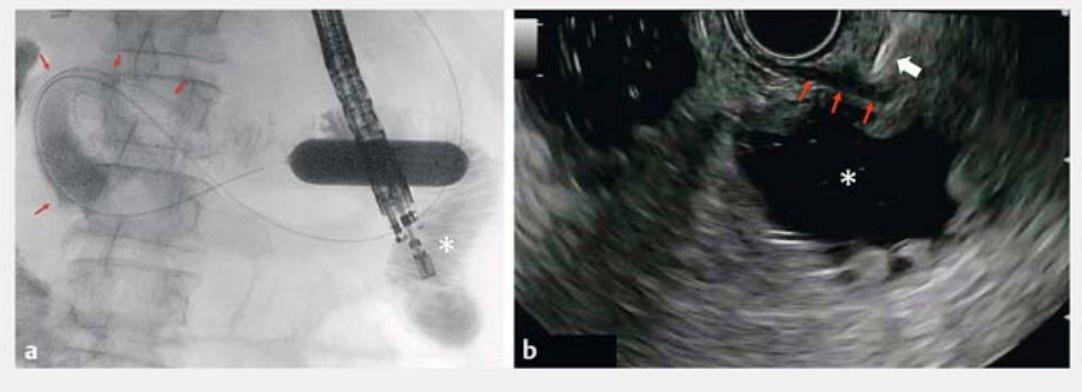

- Fig. 1 Identification of the jejunal target loop on: a fluoroscopic view, showing a 20-mm dilation balloon passed through the duodenal stent (red arrows) and the target loop $\left({ }^{*}\right)$ with contrast medium inside it; $\mathbf{b}$ endoscopic ultrasound view, showing the target loop with liquid inside $\left({ }^{*}\right)$ in close apposition to the gastric wall (red arrows). The white arrow indicates the tip of the electrocautery-enhanced lumen-apposing metal stent delivery system.

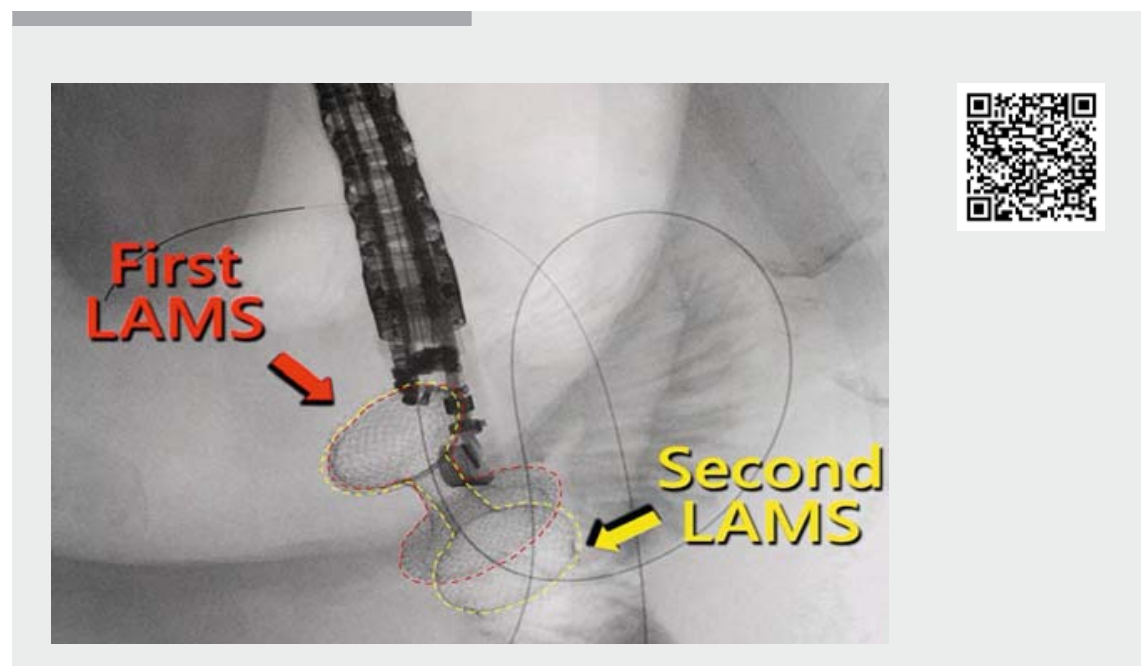

$\checkmark$ Video 1 Video showing the lumen-apposing metal stent (LAMS)-in-LAMS rescue technique for salvaging a misdeployed LAMS during an endoscopic ultrasound-guided gastroenterostomy. The second LAMS (yellow dashed line) was released through the first misdeployed LAMS (red dashed line) on a preserved wire access to the target loop.

LAMS-in-LAMS technique is an effective procedure for salvaging a misdeployed LAMS during EUS-GE, assuring correct lumen apposition between the stomach and jejunum without need for any further device.

Endoscopy_UCTN_Code_CPL_1AL_2AG

\section{Competing interests}

None 

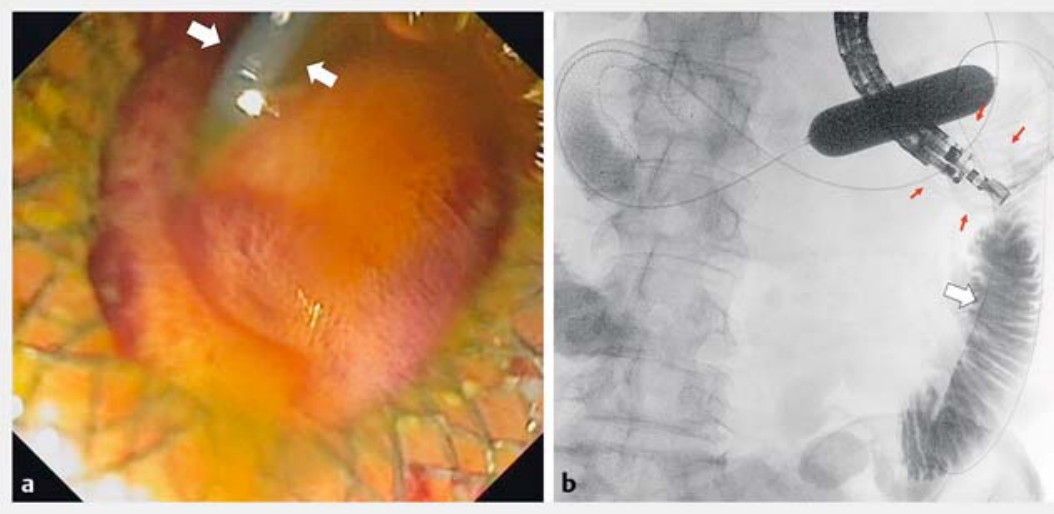

- Fig. 2 The deployed lumen-apposing metal stent (LAMS) with the dislodged distal flange. a Endoscopic view of the proximal flange of the LAMS in the gastric antrum correctly in place. The outer wall of the target loop is visible with the guidewire in place (white arrows). b Fluoroscopic view showing the deployed LAMS (red arrows) with the distal flange released outside of the target loop and the guidewire in place (white arrow).

The authors

Dario Ligresti, Michele Amata, Luca Barresi, Antonino Granata, Mario Traina, Ilaria Tarantino

Endoscopy Service, Department of Diagnostic and Therapeutic Services, IRCCS-ISMETT (Istituto Mediterraneo per i Trapianti e Terapie ad alta specializzazione), Palermo, Italy

\section{Corresponding author}

\section{Dario Ligresti, MD}

Endoscopy Service, Department of Diagnostic and Therapeutic Services, IRCCS ISMETT-UPMC, Via E. Tricomi 5, 90127

Palermo, Italy

Fax: +39-091-2192400

dligresti@ismett.edu

\section{References}

[1] Tyberg A, Perez-Miranda M, Sanchez-Ocaña $R$ et al. EUS-guided gastrojejunostomy with a lumen-apposing metal stent: a multicenter, international experience. Endosc Int Open 2016; 04: E276-E281

[2] Chen YI, Itoi T, Baron TH et al. EUS-guided gastroenterostomy is comparable to enteral stenting with fewer re-interventions in malignant gastric outlet obstruction. Surg Endosc 2017; 31: 2946-2952

[3] Khashab MA, Bukhari M, Baron TH et al. International multicenter comparative trial of endoscopic ultrasonography-guided gastroenterostomy versus surgical gastrojejunostomy for the treatment of malignant gastric outlet obstruction. Endosc Int Open 2017; 5 E275-E281

[4] Tyberg A, Saumoy M, Kahaleh M. Using NOTES to salvage a misdeployed lumenapposing metal stent during an endoscopic ultrasound-guided gastroenterostomy. Endoscopy 2017; 49: 1007-1008

[5] Tyberg A, Zerbo S, Barthet M et al. A novel technique for salvaging a dislodged lumenapposing metal stent during creation of an endoscopic gastrojejunostomy. Gastrointest Endosc 2016; 83: 254

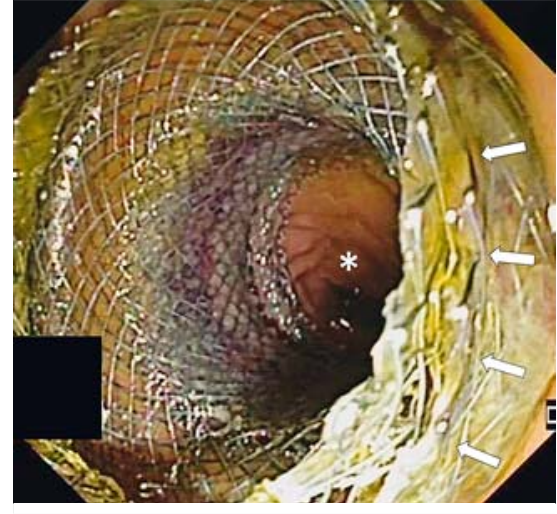

- Fig. 3 Endoscopic view of the second lumen-apposing metal stent (LAMS) correctly deployed through the first LAMS (arrows). Jejunal mucosal folds are visible $\left({ }^{*}\right)$ through the LAMS-in-LAMS system.

\section{Bibliography}

DOI https://doi.org/10.1055/a-0924-5408

Published online: 4.6.2019

Endoscopy 2019; 51: E331-E332

(c) Georg Thieme Verlag KC

Stuttgart · New York

ISSN 0013-726X

\section{ENDOSCOPY E-VIDEOS}

https://eref.thieme.de/e-videos

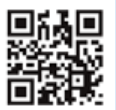

Endoscopy E-Videos is a free access online section, reporting on interesting cases and new

techniques in gastroenterological endoscopy. All papers include a high quality video and all contributions are freely accessible online.

This section has its own submission website at

https://mc.manuscriptcentral.com/e-videos 